\title{
Chronic restraint stress decreases the repair potential from mesenchymal stem cells on liver injury by inhibiting TGF- $\beta 1$ generation
}

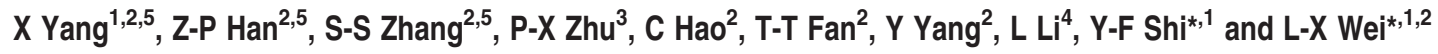

Chronic psychological stress has been demonstrated to play an important role in several severe diseases, but whether it affects disease therapy or not remains unclear. Mesenchymal stem cells (MSCs) have been demonstrated to have therapeutic potentials in treating tissue injury based on their multidifferentiation potential toward various cell types. We investigated the effect of chronic restraint stress on therapeutic potential of MSCs on carbon tetrachloride $\left(\mathrm{CCl}_{4}\right)$-induced liver injury in mice. $\mathrm{CCl}_{4^{-}}$ induced mice were injected with enhanced green fluorescent protein-MSCs, which was followed by chronic restraint stress administration. Corticosterone and RU486, a glucocorticoid receptor (GR) antagonist, were employed in vivo and in vitro, too. In the present study, we illustrated that MSCs could repair liver injury by differentiating into myofibroblasts (MFs) which contribute to fibrosis, whereas stress repressed differentiation of MSCs into MFs displayed by reducing $\alpha$-smooth muscle actin ( $\alpha$-SMA, a solid marker of MFs) expression. Whereas RU486 could maintain the liver injury reduction and liver fibrosis increases induced by MSCs in stressed mice and block the decrease of $\alpha$-SMA expression induced by stress. Furthermore, chronic stress inhibited MFs differentiation from MSCs by inhibiting transforming growth factor- $\beta 1$ (TGF- $\beta 1$ )/Smads signaling pathway which is essential for MFs differentiation. Chronic stress reduced autocrine TGF- $\beta 1$ of MSCs, but not blunted activation of Smads. All these data suggested that corticosterone triggered by chronic stress impaired liver injury repair by MSCs through inhibiting TGF- $\beta 1$ expression which results in reduced MFs differentiation of MSCs.

Cell Death and Disease (2014) 5, e1308; doi:10.1038/cddis.2014.257; published online 26 June 2014

Liver fibrosis is a wound repairment event in response to chronic injuries induced by a series of causes, such as viral hepatitis infection, alcohol, drugs, autoimmune reaction and metabolic diseases, ${ }^{1}$ which is characterized by excessive deposition of extracellular matrix proteins. If liver injury could not be repaired in time, the fibrosis would continue and come to a bad cycle that will alter the balance of matrix secretion and degradation. Cirrhosis, the end stage of fibrosis, mortality rate which increases speedily worldwide ${ }^{2}$ appears to be a large health burden in the world. There is still no effective and feasible treatment of cirrhosis apart from orthotopic liver transplantation. ${ }^{3}$ Therefore, treating liver injury at early stage seems to be crucial to arrest cirrhosis progression. In general, some factors resulting in liver injury could not be removed; hence, alternative strategies to repair liver injury at early stage needs to be developed.

With the growing enthusiasm of stem cell therapy, the application of mesenchymal stem cells (MSCs) on liver injury repair attracts more and more attention. In the trend of stem cell therapy, there are still unresolved problems in clinical application, such as the risk of teratoma formation, ethical issue, heterogeneity rejection and normalized production. However, MSCs become the most promising candidates for treatment in recent years because they are free of ethical concerns, without the risk of teratoma formation, and with low immunogenicity. MSCs have been isolated from a wide array of tissues successfully ${ }^{4-15}$ and can be cultured in vitro. Dependent on the nature of injurytropism and multipotent differentiation capacity, they have been shown to be highly effective to treat various tissue injury and degenerative diseases, such as myocardial infarction, liver cirrhosis, spinal cord injury, bone damage, cornea damage, burn-induced skin defects and other tissue injuries. ${ }^{16}$ There have been reports demonstrating that exogenous MSCs can repair damaged liver, but the mechanisms are diverse. ${ }^{17,18}$

It has been reported that myofibroblasts (MFs) are activated and contribute to wound healing after tissue injury. ${ }^{19}$ Hepatic stellate cells are not the only sources of MFs, ${ }^{20}$ as MSCs can

\footnotetext{
${ }^{1}$ Key Laboratory of Stem Cell Biology, Institute of Health Sciences, Shanghai Jiao Tong University School of Medicine (SJTUSM) and Shanghai Institutes for Biological Sciences (SIBS), Chinese Academy of Sciences (CAS), Shanghai, China; ${ }^{2}$ Tumor Immunology and Gene Therapy Center, Eastern Hepatobiliary Surgery Hospital, the Second Military Medical University, Shanghai, China; ${ }^{3}$ Department of Pharmacy, Chang Hai Hospital, the Second Military Medical University, Shanghai, China and ${ }^{4}$ Department of Anesthesia, Intensive Care Units, Eastern Hepatobiliary Surgery Hospital, the Second Military Medical University, Shanghai, China

${ }^{*}$ Corresponding author: Y-F Shi, Key Laboratory of Stem Cell Biology, Institute of Health Sciences, Shanghai Jiao Tong University School of Medicine and Shanghai Institutes for Biological Sciences, Chinese Academy of Sciences, 225 South Chongqing Road, Shanghai 200025, China. Tel: + 862163848329 ; Fax: + 8621 63852655; E-mail: shiyufang2@gmail.com

or L-X Wei, Tumor Immunology and Gene Therapy Center, Eastern Hepatobiliary Surgery Hospital, the Second Military Medical University, 225 Changhai Road, Shanghai 200438, China. Tel: + 8621 81875331; Fax: + 8621 35030398; E-mail: weilixin_smmu@163.com

${ }^{5}$ These authors contributed equally to this work.

Abbreviations: $\alpha$-SMA, $\alpha$-Smooth muscle actin; ALT, alanine aminotransferase; AST, aspartate aminotransferase; $\mathrm{CCl}_{4}$, carbon tetrachloride; EGFP, enhanced green fluorescent protein; GC, glucocorticoid; GR, glucocorticoid receptor; MSCs, mesenchymal stem cells; MFs, myofibroblasts; TGF- $\beta 1$, transforming growth factor- $\beta 1$ Received 24.1.14; revised 19.4.14; accepted 29.4.14; Edited by M Agostini
} 
differentiate into MFs too. ${ }^{21,22}$ Hence, it is suggested that MSCs repair liver injury through differentiating into MFs which is consistent with our results to some extent. In our study, exogenerous MSCs in early stage of liver injury could differentiate into MFs which contribute to liver fibrosis, and repaired liver injury in carbon tetrachloride $\left(\mathrm{CCl}_{4}\right)$-induced mouse model. Therefore, we illustrated that elevated fibrosis exerted by MSCs at early stage of liver injury could reduce liver damage, even though fibrosis at late stage of liver injury results in liver failure. Transforming growth factor- $\beta 1$ (TGF$\beta 1)$, as a known growth factor associated with liver fibrosis, was documented to be involved in MFs differentiation from stromal cell types by inducing the expression of $\alpha$-smooth muscle actin ( $\alpha$-SMA), a reliable marker of differentiated MFs. ${ }^{19,23-31}$ There has been reports demonstrating that MSCs express $\alpha$-SMA after TGF- $\beta 1$ treatment, ${ }^{24,25,32,33}$ and autocrine of TGF- $\beta 1$ from MSCs after TGF- $\beta 1$ administration has been reported too. ${ }^{33}$

During stem cell treatment, there are various factors affecting the therapy efficiency. Despite the attention paid to their own properties of MSCs, there is little consideration on the mental status of patients. Chronic stress, as a negative emotion, ${ }^{34}$ accompanies with patients and exists in the process of disease therapy. Chronic stress has an important role in the occurrence and development of various considerable diseases among cardiovascular system, digestive system, immune system and nervous system. However, the role of chronic stress in the efficiency of MSCs therapy continues to be unclear. In stress system, the hypothalamicpituitary-adrenal and the sympathetic-adrenal-medullary axises are activated, and thereby provoke the releasing of glucocorticoid (GC) (corticosterone in rodents and cortisol in humans ${ }^{35,36}$ ) and adrenal hormones, which are the main stress hormones. We are eager to know whether response to psychological stress of central nervous system influences therapeutic effect of MSCs on liver injury. In our study, mice were subjected to restraint stress after MSCs injection in $\mathrm{CCl}_{4}$-induced liver fibrosis model. Here, we demonstrated that stress repressed the function of MSCs in liver injury repair through directly affecting on MSCs.

\section{Results}

Increased liver fibrosis induced by MSCs contributed to liver injury repair, and chronic stress reversed this process. We established a $\mathrm{CCl}_{4}$-induced liver injury model in 6-8-week-old mice. To determine the role of MSCs in liver injury repair, we isolated enhanced green fluorescent protein (EGFP)-MSCs from bone marrow of EGFP transgenic mice and transplanted them into the mice injected with $\mathrm{CCl}_{4}$ intravenously. We found that when MSCs were injected at the fourth week after $\mathrm{CCl}_{4}$ intragastric administration, alanine aminotransferase (ALT) and aspartate aminotransferase (AST) levels were reduced significantly. From the data above, we could conclude that exogenerous MSCs reduced liver injury significantly. On the other hand, liver fibrosis was tested by Sirius Red staining. As shown in Figures 1c and d, compared with $\mathrm{CCl}_{4}$ group, exogenerous MSCs injection made the pseudolobules formation clear and they promoted liver fibrosis in a large extent. These data showed that injury repair is consistent with fibrosis promotion, which suggested that liver fibrosis induced by MSCs contributed to liver damage reduction.

Chronic stress, which always accompanies with patients through the process of disease, has drawn little attention in clinical therapy. In order to investigate what effect chronic stress has on MSCs repairing liver injury, restraint was used to simulate chronic stress. At the fourth day, when almost all of exogenerous MSCs have migrated into the injury sites after injection (Supplementary Figure 2A), mice were exposed to restraint stress. At the second week after the end of stress, mice were killed. As shown in Figure 1, MSCs repaired liver injury and promoted liver fibrosis, but compared with $\mathrm{CCl}_{4}+$ MSCs group, stress increased ALT and AST level and reduced liver fibrosis. The hematoxylin eosin staining also showed the same result of liver injury indicated by ALT and AST level (Figure 1e), which suggested that stress reversed the function of exogenous MSCs on liver injury repair and fibrosis.

Chronic stress inhibited MFs differentiation of MSCs. Liver fibrosis happened as an injury repair event induced by the activation of MFs. As liver is injured, not only cells such as hepatic stellate cells and fibroblasts in situ contribute to injury repair but MSCs recruited from bone marrow have also been shown to be involved in injury repair process. ${ }^{22}$ To explore how chronic stress attenuated the therapeutic effect of MSCs on liver injury, we tested the effect of chronic stress on differentiation of MSCs into MFs in vivo and in vitro. Double staining with anti-GFP and anti- $\alpha$-SMA antibodies of immunofluorescence assay was done to determine the distribution of MSCs and expression of $\alpha$-SMA. As shown in Figure 2A, MSCs recruiting to liver injury sites could differentiate into MFs determined by co-expression of EGFP and $\alpha$-SMA. However, in the MSCs plus stress group, without affecting migration of MSCs into liver injury sites (Supplementary Figure 2B) from bone marrow, the efficiency of MFs differentiation from MSCs was reduced significantly (Figures 2A and B).

To verify this result further, we detected the effect of stress on MFs differentiation from MSCs in vitro. We plated MSCs into 24 -well plates, and after cells reached $50 \%$ confluence, $2 \mathrm{ng} / \mathrm{ml}$ of TGF- $\beta 1$ and $2 \%$ serum from mice with or without stress were added. TGF- $\beta 1$ application was used as a positive control, as MSCs express $\alpha$-SMA after TGF- $\beta 1$ stimulation. Four days after treatment, the immunofluorescence assay showed that TGF- $\beta 1$ could induce expression of $\alpha$-SMA in MSCs. With the existence of TGF- $\beta 1$, serum from control mice did not alter the expression of $\alpha$-SMA compared with only TGF- $\beta 1$-treated cells. However, serum from stressed mice decreased $\alpha$-SMA expression strongly (Figure 2C), which indicated that stress repressed MFs differentiation from MSCs.

Chronic stress promoted corticosterone production. As the main stress hormone, GC has been demonstrated to be critical in neuroendocrine system, which is an important effector of hypothalamic-pituitary-adrenal axis activation. GC has also been reported to function on MSCs through bonding with GR in MSCs. ${ }^{37}$ To detect the GC secretion in 



Figure 1 Chronic stress repressed the function of exogeneous MSCs in liver injury repair and hepatic fibrosis. Mice serum was analyzed for ALT (a) and AST (b). Sirius Red staining $(\times 100)(\mathbf{c})$ and liver hydroxyproline analysis $(\mathrm{d})$ were performed to detect liver fibrosis. Hematoxylin eosin $(\mathrm{HE})$ staining was done to detect liver injury $(\times 200)\left(\right.$ e). OO, olive oil; OS, oil + stress; $\mathrm{CS}, \mathrm{CCl}_{4}+$ stress; $\mathrm{CM}, \mathrm{CCl}_{4}+\mathrm{MSCs}$; CMS, $\mathrm{CCl}_{4}+\mathrm{MSCs}+$ stress. ${ }^{*} P<0.05,{ }^{* \star} P<0.01$

mice after restraint stress, we sent the mice with or without $\mathrm{CCl}_{4}$ injection for 3 weeks to stress. Corticosterone level in serum was determined from mice with or without stress by enzyme-linked immunosorbent assay (ELISA). In the serum of stressed mice, corticosterone concentration was upregulated approximately two to threefold compared with nonstressed mice (Figure 3), immaterial of whether the mice were administrated with $\mathrm{CCl}_{4}$ or not.

Corticosterone impaired the liver injury repair by MSCs by inhibting MFs differentiation of MSCs. To verify the role of corticosterone in chronic stress on liver injury repair by MSCs, corticosterone was subcutaneously administrated into mice at the third day after MSCs injection in parallel with restraint stress in another group. We also used RU486 to block the function of stress in vivo. Results indicated that MSCs could reduce liver injury implied by decreased ALT and AST level, but corticosterone application attenuated the effect of MSCs which was consistent with stress group. Surprisingly, RU486 could downregulate ALT and AST level compared with the stress group, which indicated that RU486 could reverse the effect of stress (Figures $4 a$ and b). The liver injury extent indicated by hematoxylin eosin staining was consistent with that the ALT and AST level suggested in (Figure 4e).
Liver fibrosis analysis was performed. Fibrosis level in MSCs plus corticosterone group was reduced compared with the MSCs group, which indicated that corticosterone could reverse the promoted liver fibrosis by MSCs, which could also be observed when mice was subjected to stress. Furthermore, RU486, as a GC receptor antagonist, could block the liver fibrosis reduced by stress (Figures $4 \mathrm{c}$ and $\mathrm{d}$ ). Therefore, we concluded from all the above data that stress repressed therapeutic effect of MSCs through corticosterone production.

To assess the role of corticosterone in the MFs differentiation from MSCs, in the differentiation medium with $2 \mathrm{ng} / \mathrm{ml}$ of TGF- $\beta 1$, we added $2 \% \mathrm{~N}$ (serum from normal mice), $2 \% \mathrm{~S}$ (serum from stressed mice), 2\% S plus RU486 (100 nM) or corticosterone (200 nM), respectively. As shown in Figure 4f, corticosterone had the same effect with stress serum, which could inhibit $\alpha$-SMA expression displayed by decreased fluorescence, and RU486 reversed the reduction of $\alpha$-SMA expression induced by stress serum. Collectively, chronic stress inhibited MFs differentiation of MSCs by stimulating corticosterone secretion.

Corticosterone downregulated TGF- $\beta 1$ production by MSCs. At the initial stage of MFs differentiation, TGF- $\beta 1$ is the key-inducing factor. TGF- $\beta 1$ induces expression of $\alpha$-SMA by oligomerization of type I and type II TGF $\beta$ 
A


$\mathrm{CM}$

CMS

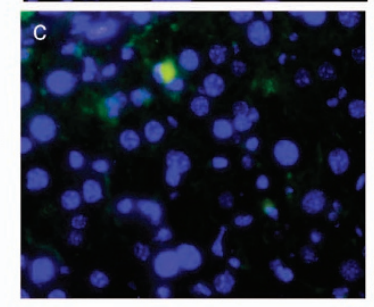

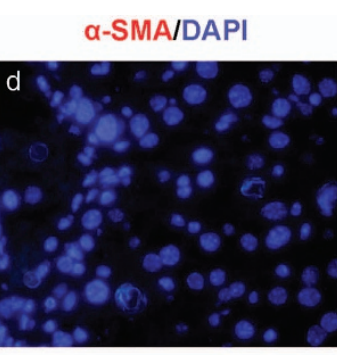
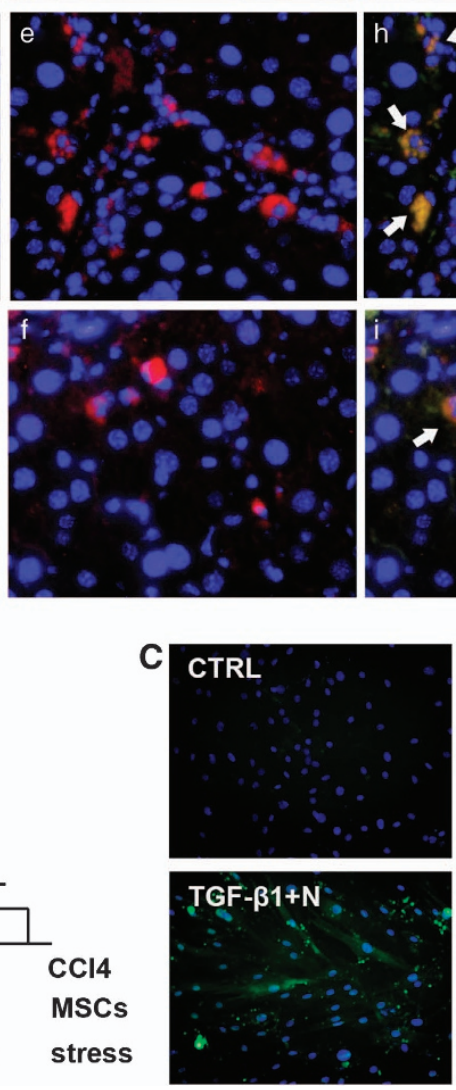
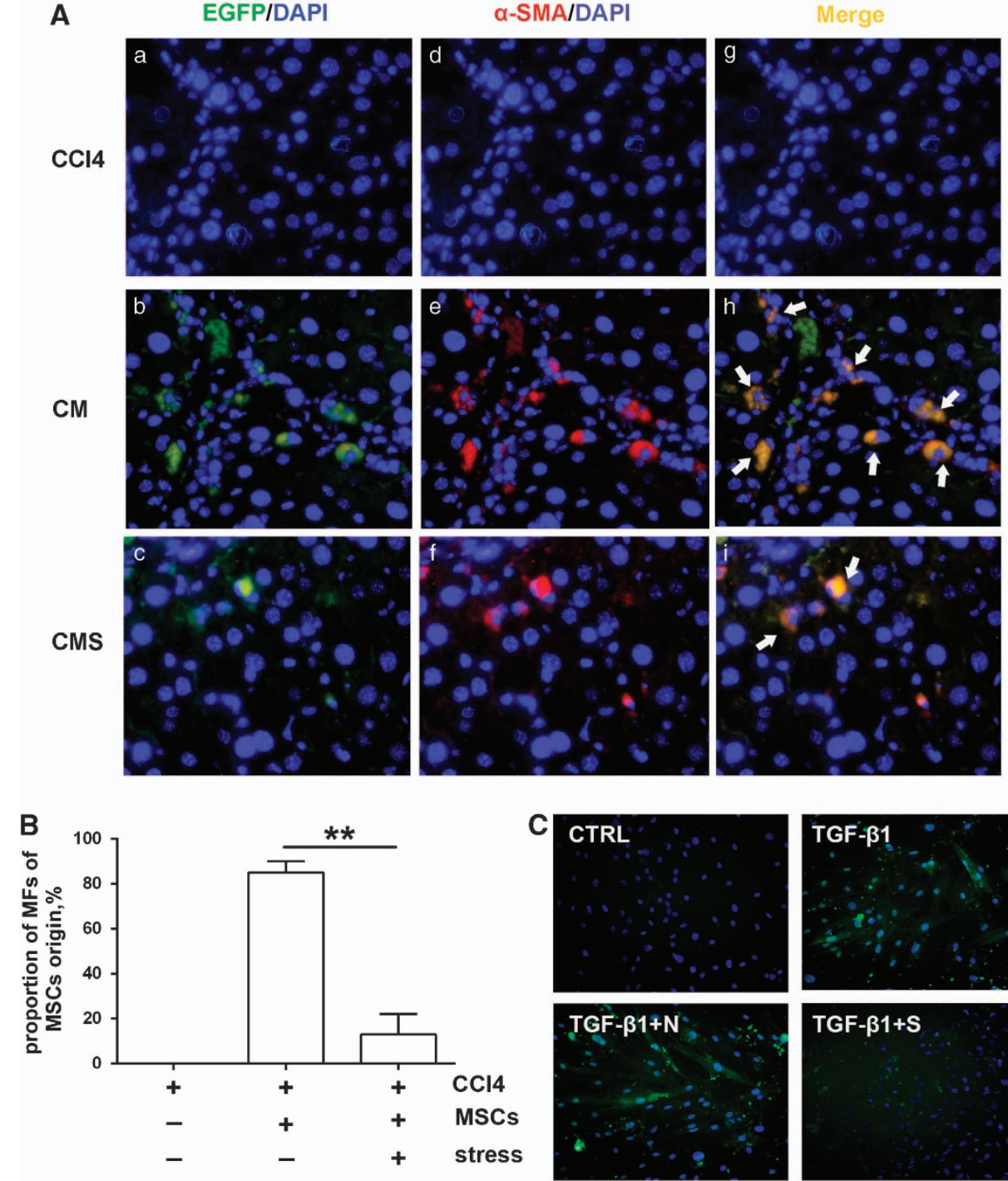

Figure 2 Chronic stress inhibited MFs differentiation of MSCs in vivo and in vitro. Liver sections were stained by immunofluorescence for MSCs (EGFP, green) and MFs $(\alpha$-SMA, red) $(\times 400)$, co-positive stands for MFs differentiation of MSCs (yellow, white arrow) (A). Percentage of co-positive cells in recruited MSCs was calculated (B). MSCs were induced to differentiate into MFs in vitro. Cytoimmunofluorescence was done to detect $\alpha$-SMA expression on MSCs (C). 4',6-diamidino-2-phenylindole (DAPI) was used to stain nuclei (blue). 00 , olive oil; $\mathrm{CM}, \mathrm{CCl}_{4}+\mathrm{MSC}$ group; $\mathrm{CMS}, \mathrm{CCl}_{4}+\mathrm{MSC} S+$ stress group. $\mathrm{N}$, serum from normal mice; $\mathrm{S}$, serum from stressed mice. ${ }^{* \star} P<0.01$

receptors and then activates Smads by phosphorylation. In order to assess whether stress alters TGF- $\beta 1$ expression, we extracted RNA from freezed liver specimens and tested mRNA expression of TGF- $\beta 1$. As shown in Figure 5a, TGF$\beta 1$ expression was upregulated in injured liver induced by $\mathrm{CCl}_{4}$, MSCs injection increased TGF- $\beta 1$ expression to a higher level. However, stress played a negative role on TGF$\beta 1$ expression. MSCs injection with subsequent stress could not promote TGF- $\beta 1$ expression compared with $\mathrm{CCl}_{4}$ group. In other words, stress repressed MSCs-associated TGF- $\beta 1$ expression increase. But stress together with RU486 could not repress the function of MSCs.

In addition, we tested TGF- $\beta 1$ expression on protein level in liver by IHC assay. Tissue sections were stained with antiTGF- $\beta 1$ antibody. Data from immunohistochemistry assay were consistent with the real-time PCR results. Interestingly, TGF- $\beta 1$ expressed at the position where MSCs were recruited
(Figure $5 b)$. To verify the effect of corticosterone on TGF- $\beta 1$ expression of MSCs, we stimulated MSCs with $2 \mathrm{ng} / \mathrm{ml} \mathrm{TGF-}$ $\beta 1$, and we added $2 \% \mathrm{~N}, 2 \% \mathrm{~S}, 2 \% \mathrm{~S}$ plus $100 \mathrm{nM}$ of RU 486 , or $200 \mathrm{nM}$ of corticosterone in the culture; $12 \mathrm{~h}$ later, medium was replaced by serum-free medium. ELISA and real-time PCR were performed after $24 \mathrm{~h}$. The data showed that MSCs could secret TGF- $\beta 1$ after TGF- $\beta 1$ stimulation and stress could inhibit TGF- $\beta 1$ expression both in extracellular secretion and intracellular mRNA expression of MSCs, and RU486 could reverse the effect of stress (Figures $5 c$ and d). $\alpha$-SMA expression was consistent with TGF- $\beta 1$ expression level (Figure 5e), which provided further evidence to support that TGF- $\beta 1$ stimulates the MFs differentiation of MSCs. To reinforce the effect of GC on TGF- $\beta 1$ production, we detected TGF- $\beta 1$ production in human liver cell line Chang Liver after cortisol treatment. As shown in Supplementary Figure 3, cortisol could inhibit TGF- $\beta 1$ production. 


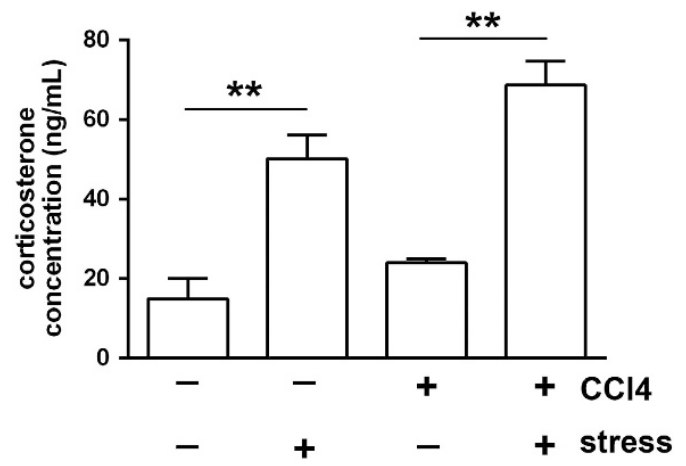

Figure 3 Corticosterone concentration in mice serum. Naive or $\mathrm{CCl}_{4}$-treated mice were stressed or not, then killed and serum was collected and detected by ELISA for corticosterone. ${ }^{* *} P<0.01$

\begin{abstract}
Activation of Smads in TGF- $\beta 1 / \mathrm{Smads}$ pathway was not affected by corticosterone directly. TGF- $\beta 1 / \mathrm{Smads}-$ dependent signal pathway is known to be critical for MFs differentiation and this pathway is activated by oligomerization of type I and type II TGF $\beta$ receptors and subsequent phosphorylation of Smad2/3. To examine whether chronic stress affects activation of Smads or not directly apart from downregulating TGF- $\beta 1$ expression, we treated MSCs with $50 \mathrm{ng} / \mathrm{ml} \mathrm{TGF}-\beta 1$, and with $2 \% \mathrm{~N}, 2 \% \mathrm{~S}, 2 \% \mathrm{~S}$ plus $100 \mathrm{nM}$ of $\mathrm{RU} 486$, or $200 \mathrm{nM}$ of corticosterone in addition for 4 days, then detected phosphorylation of Smads in MSCs by western blotting assay. As shown in Figure 6, with the same level of TGF- $\beta 1$ stimulus, stress could not inhibit phosphorylation of Smad2 and Smad3. Therefore, chronic stress only inhibited production of TGF- $\beta 1$ without affecting downstream of the TGF- $\beta 1$ signaling pathway.
\end{abstract}

\section{Discussion}

MSCs, with the presence of cell surface marker CD29, CD44, CD90, Sca1 and absence of hematopoietic antigens CD34, CD45, are a population of heterogenerous tissue stem cells, which were isolated from the bone marrow originally. In addition, they express MHC class Iow and do not express $\mathrm{MHC}$ class II, which makes them having low immunogenicity. They are characterized by the property of self-renewal, plastic adhesion and fibroblast-like morphology. They have the capacity of differentiating into multiple cell lines, such as adipocytes, osteocytes and chondrocytes, even other cell types besides mesoderm lineages. They have been shown to be isolated from a wide array of tissues, such as bone marrow, fat, skin and can be cultured in vitro. Furthermore, MSCs exhibit a strong capacity of immunomodulation and inflammation tropism. Due to their properties above and free of teratoma formation and ethical issues, they have a strong potential in clinical application.

After tissue is injured, the repair process involves two stages: a regenerative phase, injured tissues are replaced by cells of the same type; and a fibrosis phase, connective tissue replaces the normal tissue. When normal cells cannot repair the injury completely, MFs will be activated to repair injury and come to the fibrosis phase. ${ }^{19,38}$ Fibrosis contributes to damage reduction and is a result of injury repair. There is an increasing evidence showing that the hepatic fibrosis of early stage is beneficial and reversible if the stimuli are removed and injury is repaired successfully. ${ }^{39}$ Activation of MFs will be terminated and MFs will disappear by apoptosis when the tissue is repaired. ${ }^{40}$ MFs are generally considered to have key roles in physiological reconstruction of connective tissue and wound healing. $\alpha$-SMA has been accepted as a solid marker of activation of MFs. From previous reviews, we can conclude that MFs originate from various cell populations, including local tissue fibroblasts, resident hepatic stellate cells, epithelial cells undergoing epithelial-mesenchymal transition and fibroblast-like cells from bone marrow. ${ }^{21,41-48}$ However, contribution of MSCs to MFs activation is still unclear even if differentiation of MSCs into MFs has gained increasing attention. In $\mathrm{CCl}_{4}$-induced mouse liver injury model, MSCs recruited into injury sites have been demonstrated to express $\alpha$-SMA. However, whether this fate is beneficial or detrimental for liver injury repair remains to be an unresolved problem. . $^{38,49,50}$

While in clinical therapy, the efficiency of MSCs therapy is limited and varies among different patients. To explore the problem and optimize the treatment efficiency of MSCs, concerns have been focused on the quality of MSCs, including the cell viability, culture condition of cells, passage of cells and even origins where the cells come from. But there is little attention paid to the patients personally. Indeed, emotion of the patients who accepted MSCs as treatment is equally important. The fate of MSCs in recipients is responsible for the efficiency of treatment. In almost all the patients, chronic stress accompanies them through the disease progression, the negative emotion affects the development and treatment of disease. In our study, we found that chronic stress could repress the therapeutic effect of $\mathrm{MSCs}$ in $\mathrm{CCl}_{4}$-induced liver injury model. While, from this point, self-healing by endogenous MSCs can also be impaired.

Chronic stress-associated corticosterone, which is one type of steroid hormone coming from adrenal gland, has been considered to affect almost every tissue and has an important role in stress-related diseases. ${ }^{36}$ There were also evidences showing that glucocorticoid induced by stressors could repress immune responses by diminishing pro-inflammatory cytokines. $^{3}$ Interestingly, glucocorticoid has been demonstrated to have the capacity to slow wound healing. ${ }^{3}$ They explain the phenomenon as the same cause with immunosuppression. In the present study, we analyzed this problem from another aspect which is a novel standpoint. We found that stress repressed differentiation of MSCs into MFs, which resulted in impaired liver injury repair. More recently, GC has been investigated to interfere in differentiation of MSCs into adipocytes, osteoblasts, myoblasts and chondrocytes. The underlying mechanism of GC participating in the differentiation process remains unknown, apart from bonding with GR. The effects of GC on mature fibroblasts have been usually investigated in skin. Surprisingly, previous results came as the negative effect of GC on fibroblasts proliferation and the ability of synthesizing mature collagen. ${ }^{51}$ However, there has been no subtle investigation on the mechanisms of GC affecting MFs differentiation.

In the present study, corticosterone had the same effect with chronic stress on MSCs in reducing liver injury. 



C
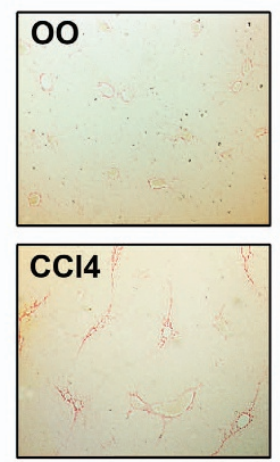
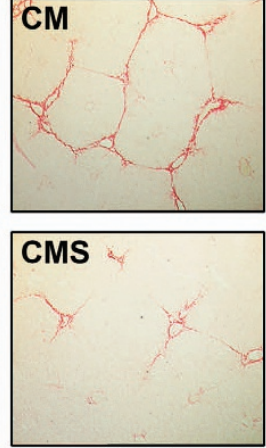

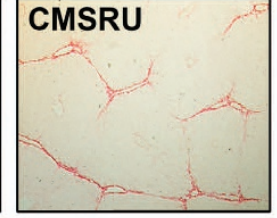

CMCor

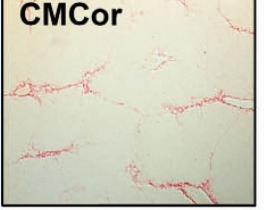

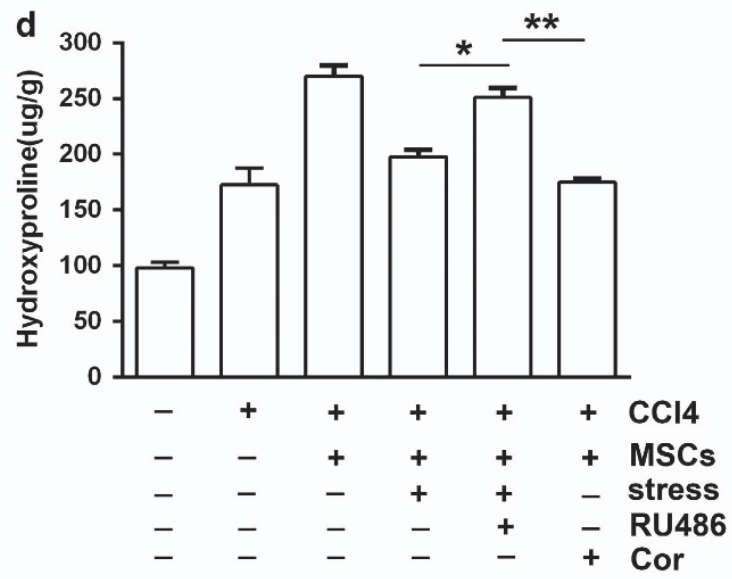

e
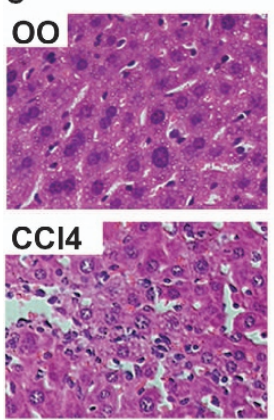
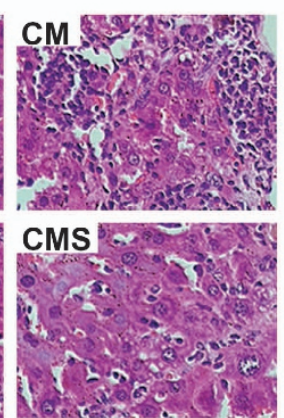


f
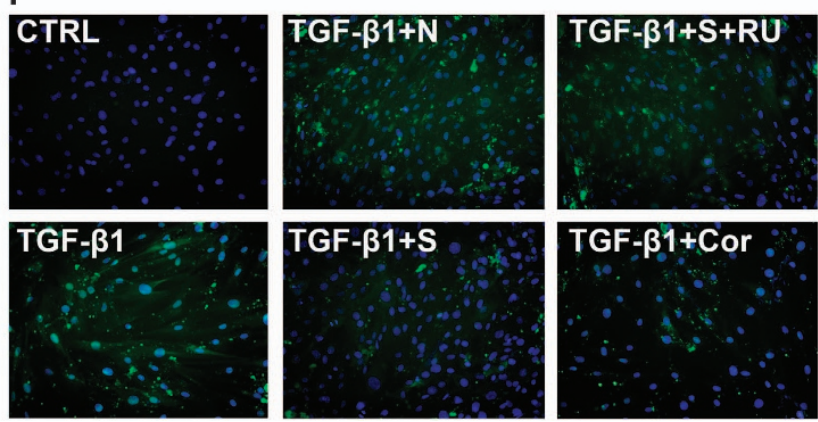

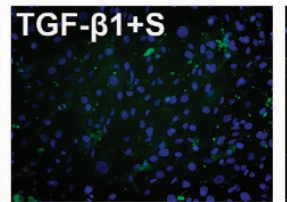

TGF- $\beta 1+$ Cor

Figure 4 Corticosterone repressed MSCs differentiation into MFs. Mice serum was collected and analyzed for ALT (a) and AST (b). Sirius Red staining ( $\times 100)$ (c) and liver hydroxyproline analysis (d) were done to detect liver fibrosis. Hematoxylin eosin (HE) staining was done to detect liver injury ( $\times 200)(\mathbf{e})$. MSCs were induced to differentiate into MFs in vitro. MFs differentiation efficiency of MSCs was determined by cytoimmunofluorescence assay (f). 4',6-diamidino-2-phenylindole (DAPI) was used to

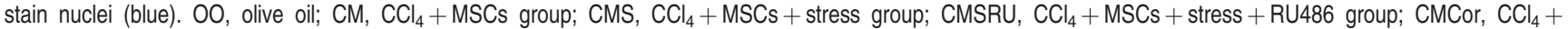
MSCs + corticosterone group; N, serum from normal mice; $\mathrm{S}$, serum from stressed mice. RU, RU486; Cor, corticosterone. * $P<0.05$, ${ }^{*} P<0.01$

In addition, RU486 could reverse the effect of chronic stress. These results suggested that chronic stress repressed liver injury repair by MSCs by producing corticosterone. Then, we addressed the problem why chronic stress-associated corticosterone attenuates MFs differentiation of MSCs for the first time. In vivo and in vitro experiments both showed that corticosterone reduced the production of TGF- $\beta 1$. Interestingly, immunohistochemistry assay showed that TGF- $\beta 1$ was expressed in the injury sites where MSCs were recruited. Whereas, after corticosterone or chronic stress was administrated to mice, TGF- $\beta 1$ expression was reduced. In vitro assay showed that corticosterone could block autocrine and intracellular expression of TGF- $\beta 1$ in MSCs, which is compatible to the in vivo assay. Both the in vivo and in vitro results suggested that chronic stress-reduced expression of TGF- $\beta 1$ was associated with the reduction of TGF- $\beta 1$ generation from MSCs. To elucidate whether corticosterone has a negative role in TGF- $\beta 1$ production through interacting with GR, we used RU486 in vivo and in vitro; data showed that RU486 could block the function of corticosterone, which 
a

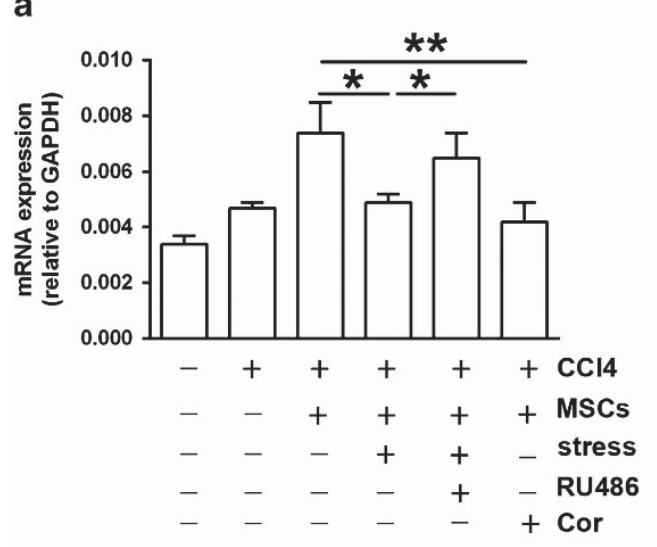

$b$
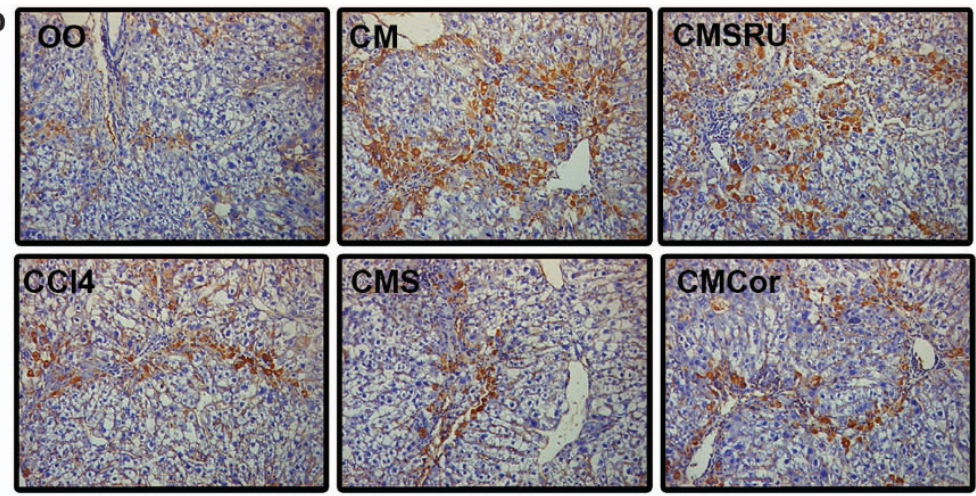

C

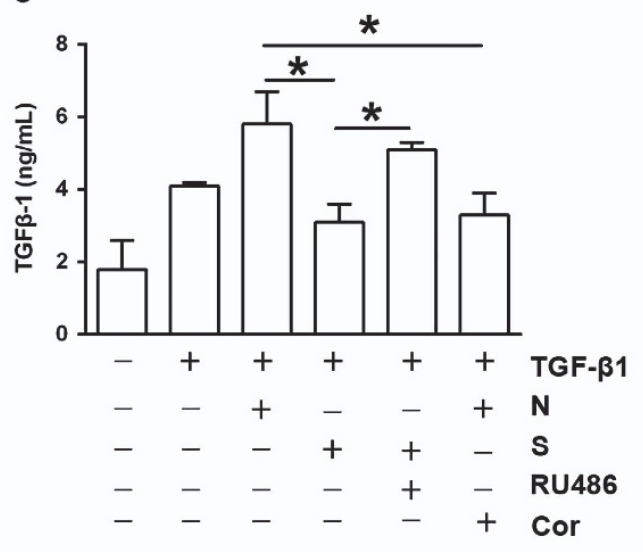

d

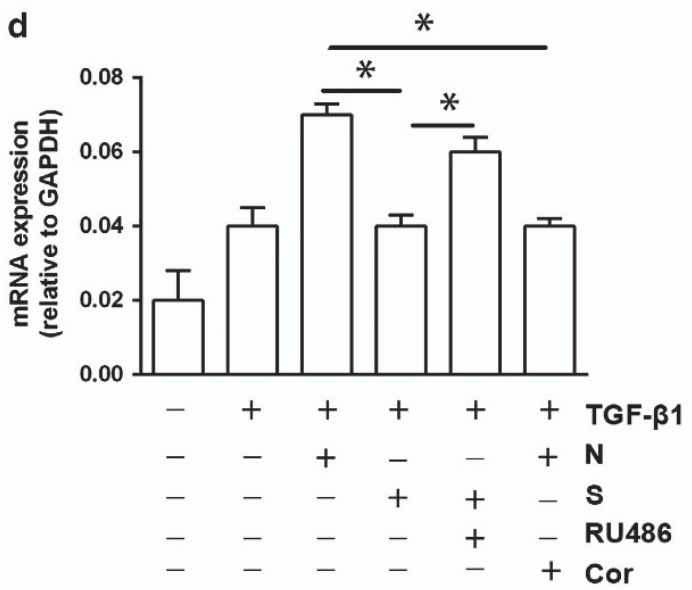

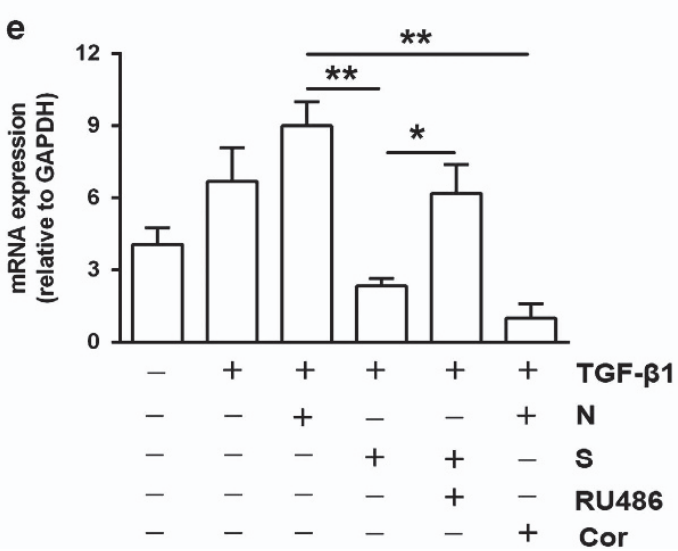

Figure 5 Corticosterone inhibited TGF- $\beta 1$ expression in livers and MSCs. TGF- $\beta 1$ expression level in livers was detected by real-time PCR (a) and immunohistochemistry (b). Conditioned medium from MSCs under different conditions was collected and tested by ELISA (c). mRNA expression of TGF- $\beta 1$ (d) and corresponding $\alpha$-SMA expression in MSCs were tested by real-time PCR (e). O0, olive oil; CM, $\mathrm{CCl}_{4}+\mathrm{MSC}$ group; $\mathrm{CMS}, \mathrm{CCl}_{4}+\mathrm{MSC}$ + stress group; $\mathrm{CMSRU}, \mathrm{CCl}_{4}+\mathrm{MSCs}+$ stress $+\mathrm{RU} 486$ group; $\mathrm{CMCor}, \mathrm{CCl}_{4}+\mathrm{MSC}$ + corticosterone group; N, serum from normal mice; S, serum from stressed mice. Cor, corticosterone. ${ }^{*} P<0.05,{ }^{* *} P<0.01$

suggested that corticosterone functioned through the GC/GR pathway.

TGF- $\beta 1$ is the crucial factor in MFs differentiation ${ }^{3,33}$ and it induces $\alpha$-SMA expression through the TGF- $\beta 1 /$ Smads pathway involving phosphorylation of Smad2/3. With the same concentration of TGF- $\beta 1$ inducing MFs differentiation of MSCs, additional stress serum or corticosterone in the culture medium had no effect on the phosphorylation of Smad2/3 and expression of $\alpha$-SMA. With the same level of TGF- $\beta 1$, the bonding of TGF- $\beta 1$ with its receptors and activation of Smads could not be altered by corticosterone. These results suggested that corticosterone inhibited MFs differentiation by preventing TGF- $\beta 1$ generation, without inhibiting the activation of downstream of the TGF- $\beta 1$ pathway directly. And how corticosterone reduces production of TGF- $\beta 1$ needs further study.

Taken together, our study demonstrated that chronic stress repressed liver injury repair by MSCs by increasing 


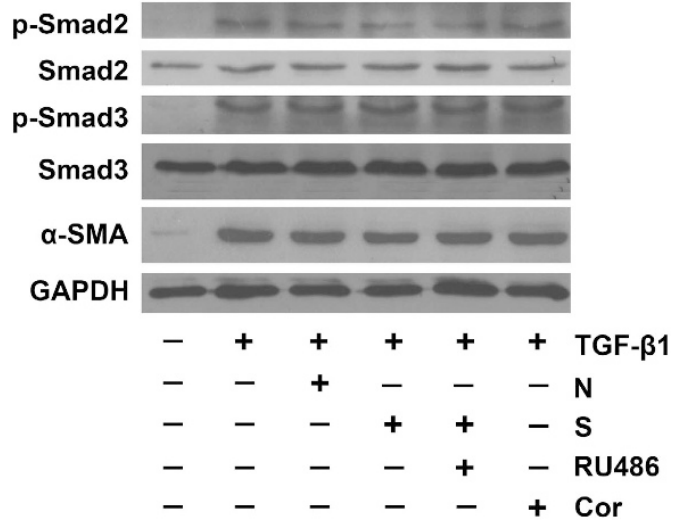

Figure 6 Corticosterone did not affect downstream of the TGF- $\beta 1 / \mathrm{Smads}$ pathway. Expression of Smad2, p-Smad2, Smad3, p-Smad3, $\alpha$-SMA was tested in MSCs by western blotting assay. Glyceraldehyde-3-phosphate dehydrogenase (GAPDH) was used as internal reference. N, serum from normal mice; S, serum from stressed mice. Cor, corticosterone

corticosterone in mice, which resulted in inhibition of MFs differentiation from MSCs. TGF- $\beta 1$ production is one target of corticosterone in inhibiting MFs differentiation without affecting downstream of the TGF- $\beta 1 /$ Smads signaling pathway. On the other hand, RU486 may contribute to increasing efficiency of MSCs in liver injury repair in stressful patients. Further research should be done to discover a safe and efficient candidate to ensure the function of MSCs. Importantly, the mental state of patients under treatment needs more attention.

\section{Materials and Methods}

Materials. Dulbecco's modified eagle medium (low glucose), fetal bovine serum, glutamax (catalog number: 35050-061), penicillin-streptomycin (catalog number:15140-122), trypsin-EDTA (catalog number: 15400-054) and fibroblast growth factor basic (catalog number: PMG0035) were purchased from Gibco (Grand Island, NY, USA). Hydroxyproline detection kit (catalog number: A030-2) was purchased from Nanjing Jiancheng Bioengineering Institute (Nanjing, China). Recombinant mouse TGF- $\beta 1$ (catalog number: 7666-MB-005) was purchased from R\&D system (Minneapolis, MN, USA). Mouse TGF- $\beta 1$ ELISA kit was purchased from Shanghai Hengyuan Biotechnology (Shanghai, China). Corticosterone ELISA kit (catalog number: ADI-900-097) was purchased from Enzo Life Sciences (Farmingdale, NY, USA). Corticosterone (catalog number: 46148), cortisol (catalog number: C-113), RU486 (catalog number: M8046) were purchased from sigma (St. Louis, MO, USA). Anti- $\alpha$ SMA antibody (catalog number: ab5694) was purchased from Abcam (Cambridge, UK). GFP antibody (catalog number: NB100-1770) was purchased from Novus Biologicals (Littleton, CO, USA). Alexa Fluor 568 goat anti-rabbit $\lg G(H+L)$ (catalog: A-11011), Alexa Fluor488 donkey anti-goat $\lg \mathrm{G}(\mathrm{H}+\mathrm{L})$ (catalog number: A-11055) were purchased from Invitrogen (Carlsbad, CA, USA). Smad2/3 antibody (catalog number:5678S), phosphate-smad2/3 rabbit $\mathrm{mAb}$ (catalog number: $8828 \mathrm{~S}$ ) were purchased from Cell Signaling Technology (Danvers, MA, USA). Anti-TGF- $\beta 1$ antibody (catalog number: ab92486) was purchased from Abcam.

Isolation and culture of mouse MSCs. Male EGFP transgenic mice aged 6-8 weeks were used to isolate bone marrow MSCs. MSCs were isolated and cultured as described by Heng Zhu et al. ${ }^{52}$ Mice were killed by cervical dislocation and the limbs were removed. We flushed bone marrow cells from the medullary cavities from the tibias and femurs with physiological saline by using a 2-ml needle. Derived bone marrow cells were suspended in single-cell suspension and seeded in DMEM medium (low glucose) with $10 \%$ fetal bovine serum, $1 \times$ glutamax, $1 \times$ penicillin-streptomycin by $1 \times 10^{7}$ cells $/ \mathrm{ml}$. Three days later, suspended cells were removed and medium was replaced with fresh medium. Thereafter, medium was replaced every three days. At the seventh day after isolation, attached MSCs were removed by trypsin-EDTA, then resuspended in fresh medium. After cells were passaged for three times, they were taken as purified MSCs and identified by adipocytes and osteoblasts differentiation (see Supplementary Figure 1). ${ }^{53}$ In the following culture of MSCs, fibroblast growth factor basic was added in a concentration of $0.5 \mathrm{ng} / \mathrm{ml}$.

Chronic restraint stress procedure. Restraint stress model was based on previous studies. ${ }^{54-56}$ Mice were assigned into home cage control or the restraint tubes ( $50 \mathrm{ml}$ conical tubes containing many holes) to limit their actions for $12 \mathrm{~h} /$ day for three consecutive days. They were put into tubes at $2000-2100 \mathrm{~h}$ with five mice per group, and released at 0800-0900 h next day. In the tubes, they could not move freely, couldn't get water and food, but they were not pressed and could breath freely.

Mouse model of liver fibrosis and treatment. In order to induce chronic liver injury, male C57BL/6 mice aged 6-8 weeks were feeded with $20 \%$ (v/v, dissolved in olive oil) $\mathrm{CCl}_{4}$ by intragastric method at a concentration of $5.0 \mathrm{ml} / \mathrm{kg}$, twice a week. At the fourth week, EGFP-MSCs $\left(1 \times 10^{6}\right.$ per mouse $)$ or saline was injected through the tail vein. Cell transplantation mice were divided into four groups: control group, without any treatment; stress group, mouse were administrated with stress at the fourth day after MSCs injection and in the following week; stress plus RU486 group, RU486 was administrated subcutaneously at a dosage of $25 \mathrm{mg} / \mathrm{kg}$ one day before the beginning of the stress sessions, thereafter RU486 was injected $2 \mathrm{~h}$ before stress period; and corticosterone group, corticosterone was injected subcutaneously at a dosage of $5 \mathrm{mg} / \mathrm{kg}$ once a day in parallel with the stress treatment. In addition, we had olive oil and stress group as control. Mice were killed at the sixth week after liver injury induction.

Liver injury analysis. Serum of mice was collected by centrifugation at $3000 \times g, 4^{\circ} \mathrm{C}$ for 10 min with anticoagulants. The ALT and AST were analyzed with a biochemical analyzer.

Hematoxylin and eosin staining. Mouse liver samples were obtained after killing and fixed in $4 \%$ paraformaldehyde, and then embedded in paraffin. In total, $5-\mu \mathrm{m}$-thick sections were prepared for the experiments. Mouse liver sections embedded in paraffin were stained with hematoxylin and eosin according to the manufacturer's protocol.

Liver fibrosis analysis. To detect liver fibrosis, the paraffin sections were stained with Sirius Red. To detect liver fibrosis quantitatively, fresh liver tissues were tested for hydroxyproline using hydroxyproline detection kit.

Immunohistochemistry and immunofluorescence staining. Immunohistochemistry assay was performed according to method described before, ${ }^{33}$ anti- $\alpha$-SMA antibody, anti-GFP antibody, anti-TGF- $\beta 1$ antibody were used. All of these antibodies were used at a dilution of $1 / 100$. Immunofluorescence and horseradish peroxidase-conjugated secondary antibodies were used correspondingly.

Real-time PCR. Total RNA from cultured cells or freezed liver tissues was extracted by trizol method. In total, $1 \mu \mathrm{g}$ RNA in $10 \mu \mathrm{l}$ for all reagents was transcripted to CDNA using bestar qPCR RT kit. The real-time PCR was performed using bestar real-time PCR master mix with an ABI Prism 7300 system (Grand Island, NY, USA). The primers of mouse genes for real-time PCR were as follows: $\alpha$-SMA, sense, $5^{\prime}$-ATGCTCCCAGGGCTGTTTT- $3^{\prime}$, and antisense, $5^{\prime}$-TTCCAACC ATTACTCCCTGATGT-3'; TGF- $\beta 1$, sense, $5^{\prime}$-CTCCCGTGGCTTCTAGTGC- $3^{\prime}$, and antisense, 5'-GCCTTAGTTTGGACAGGATCTG-3'; glyceraldehyde-3-phosphate dehydrogenase, sense, 5'-AGGTCGGTGTGAACGGATTTG-3'; and antisense, $5^{\prime}$-TGTAGACCATGTAGTTGAGGTCA-3'. The primers of human genes for real-time PCR were as follows: TGF- $\beta 1$, sense, $5^{\prime}$-CGAGCCTGAGGCCGACTAC- $3^{\prime}$; and antisense, 5'-AGATTTCGTTGTGGGTTTCCA-3'; glyceraldehyde-3-phosphate dehydrogenase, sense, 5'-CCAGGAAATGAGCTTGAGAAAGT-3'; and antisense, $5^{\prime}$-CCCACTCCTCCACCTTTGAC-3'.

ELISA. Serum from mice of control, restraint stress, $\mathrm{CCl}_{4}$-treated and $\mathrm{CCl}_{4}$ plus stress-treated mice was assayed for corticosterone with a corticosterone ELISA kit according to the manufacturer's recommendation. MSCs were treated by corresponding reagents for $12 \mathrm{~h}$, then medium was replaced by fresh 
serum-free culture medium and cells were cultured for another $24 \mathrm{~h}$, then supernatant was collected as conditioned medium and tested for TGF- $\beta 1$ by a TGF- $\beta 1$ ELISA kit.

Western blotting assay. MSCs treated with appropriate conditions were lysed in lysis buffer. The lysates were qualified using BCA kit and boiled with loading buffer for $10 \mathrm{~min}$. Thereafter, denatured protein was segregated by SDSpolyacrylamide gel electrophoresis, and then transferred onto nitrocellulose membrane. The membranes were blocked by $5 \%$ nonfat milk, and then immunoblotted by various primary monoclonal antibodies. The horseradish peroxidase-conjugated goat anti-rabbit secondary antibody and ECL were used to examine the expression of protein. Glyceraldehyde-3-phosphate dehydrogenase was used as internal conference. Expression of target proteins was normalized to glyceraldehyde-3-phosphate dehydrogenase before comparing among different groups.

Statistical analyses. Results were presented as means \pm S.E.M. and the statistical differences were analyzed by Student's $t$-test, $n=3(P<0.05$ were considered significant).

\section{Conflict of Interest}

The authors declare no conflict of interest.

Acknowledgements. This project was supported by Key Basic Research Project of China (Grant No. 2012CBA01303, 2011CB966200, 2010CB945600 2011CB965100); Key project of National Natural Science Foundation of China (Grant no 81030041); National Natural Science Foundation of China (Grant No. 31171321, 81101622, 81372330, 81101830); Special Funds for National key Sci-Tech Special Project of China (Grant No. 2012ZX10002-016, 2012ZX10002011-011); Shanghai Science and Technology Committee (Grant No. 11ZR1449500, 12ZR1439800); Shanghai Municipal Health Bureau (Grant No. 20114004) and Science Fund for Creative Research Groups, NSFC, China (Grant No. 81221061).

\section{Author contributions}

$\mathrm{XY}, \mathrm{Z}-\mathrm{PH}$ and S-SZ participated in the design and performance of the study. $\mathrm{XY}$ carried out cell culture, molecular studies and analyzed the data. XY and P-XZ did the mouse experiments. YY made the tissue sections. L-XW conceived this study and participated in its design and coordination. The manuscript was drafted by XY, revised by S-SZ and reviewed by all authors. All authors approved the final version of the manuscript to be published.

1. Abdel Aziz MT, Atta HM, Mahfouz S, Fouad HH, Roshdy NK, Ahmed HH et al. Therapeutic potential of bone marrow-derived mesenchymal stem cells on experimental liver fibrosis. Clin Biochem 2007; 40: 893-899.

2. Henderson NC, Iredale JP. Liver fibrosis: cellular mechanisms of progression and resolution. Clin Sci (Lond) 2007; 112: 265-280.

3. Glaser R, Kiecolt-Glaser JK. Stress-induced immune dysfunction: implications for health Nat Rev Immunol 2005; 5: 243-251.

4. Xu X, Zhang X, Wang S, Qian H, Zhu W, Cao H et al. Isolation and comparison of mesenchymal stem-like cells from human gastric cancer and adjacent non-cancerous tissues. J Cancer Res Clin Oncol 2011; 137: 495-504.

5. Lyahyai J, Mediano DR, Ranera B, Sanz A, Remacha AR, Bolea R et al. Isolation and characterization of ovine mesenchymal stem cells derived from peripheral blood. BMC Vet Res 2012; 8: 169

6. Li XY, Zheng ZH, Guo J, Zhang Y, Li H, Wang YW et al. Treatment of foot disease in patients with type 2 diabetes mellitus using human umbilical cord blood mesenchymal stem cells: response and correction of immunological anomalies. Curr Pharm Des 2013; 19 : 4893-4899.

7. Heo JS, Choi SM, Kim HO, Kim EH, You J, Park T et al. Neural transdifferentiation of human bone marrow mesenchymal stem cells on hydrophobic polymer-modified surface and therapeutic effects in an animal model of ischemic stroke. Neuroscience 2013; 238: 305-318.

8. Lonne M, Lavrentieva A, Walter JG, Kasper C. Analysis of oxygen-dependent cytokine expression in human mesenchymal stem cells derived from umbilical cord. Cell Tissue Res 2013; 353: 117-122

9. Vellasamy S, Sandrasaigaran P, Vidyadaran S, George E, Ramasamy R. Isolation and characterisation of mesenchymal stem cells derived from human placenta tissue. World $\mathrm{J}$ Stem Cells 2012; 4: 53-61.
10. Roubelakis MG, Tsaknakis G, Pappa KI, Anagnou NP, Watt SM. Spindle shaped human mesenchymal stem/stromal cells from amniotic fluid promote neovascularization. PLoS One 2013; 8: e54747.

11. Villanueva S, Carreno JE, Salazar L, Vergara C, Strodthoff R, Fajre F et al. Human mesenchymal stem cells derived from adipose tissue reduce functional and tissue damage in a rat model of chronic renal failure. Clin Sci (Lond) 2013; 125: 199-210.

12. Spitzer TL, Rojas A, Zelenko Z, Aghajanova L, Erikson DW, Barragan F et al. Perivascular human endometrial mesenchymal stem cells express pathways relevant to self-renewal, lineage specification, and functional phenotype. Biol Reprod 2012; 86: 58

13. Ge S, Mrozik KM, Menicanin D, Gronthos S, Bartold PM. Isolation and characterization of mesenchymal stem cell-like cells from healthy and inflamed gingival tissue: potential use for clinical therapy. Regen Med 2012; 7: 819-832.

14. Kisiel AH, McDuffee LA, Masaoud E, Bailey TR, Esparza Gonzalez BP, Nino-Fong R. Isolation, characterization, and in vitro proliferation of canine mesenchymal stem cells derived from bone marrow, adipose tissue, muscle, and periosteum. Am J Vet Res 2012; 73: $1305-1317$.

15. Mensing N, Gasse H, Hambruch N, Haeger JD, Pfarrer C, Staszyk C. Isolation and characterization of multipotent mesenchymal stromal cells from the gingiva and the periodontal ligament of the horse. BMC Vet Res 2011; 7: 42.

16. Wei X, Yang X, Han ZP, Qu FF, Shao L, Shi YF. Mesenchymal stem cells: a new trend for cell therapy. Acta Pharmacol Sin 2013; 34: 747-754.

17. Puglisi MA, Tesori V, Lattanzi W, Piscaglia AC, Gasbarrini GB, D'Ugo DM et al. Therapeutic implications of mesenchymal stem cells in liver injury. J Biomed Biotechnol 2011; 2011: 860578.

18. Du Z, Wei C, Cheng K, Han B, Yan J, Zhang M et al. Mesenchymal stem cell-conditioned medium reduces liver injury and enhances regeneration in reduced-size rat liver transplantation. J Surg Res 2013; 183: 907-915.

19. Hinz B, Phan SH, Thannickal VJ, Galli A, Bochaton-Piallat ML, Gabbiani G The myofibroblast: one function, multiple origins. Am J Pathol 2007; 170: 1807-1816.

20. Parola M, Marra F, Pinzani M. Myofibroblast-like cells and liver fibrogenesis: emerging concepts in a rapidly moving scenario. Mol Aspects Med 2008; 29: 58-66.

21. Forbes SJ, Russo FP, Rey V, Burra P, Rugge M, Wright NA et al. A significant proportion of myofibroblasts are of bone marrow origin in human liver fibrosis. Gastroenterology 2004; 126: 955-963.

22. Russo FP, Alison MR, Bigger BW, Amofah E, Florou A, Amin F et al. The bone marrow functionally contributes to liver fibrosis. Gastroenterology 2006; 130: 1807-1821.

23. Shangguan L, Ti X, Krause U, Hai B, Zhao Y, Yang Z et al. Inhibition of TGF-beta/Smad signaling by BAMBI blocks differentiation of human mesenchymal stem cells to carcinoma-associated fibroblasts and abolishes their protumor effects. Stem Cells 2012; 30: 2810-2819.

24. Yang L, Chang N, Liu X, Han Z, Zhu T, Li C et al. Bone marrow-derived mesenchymal stem cells differentiate to hepatic myofibroblasts by transforming growth factor-beta1 via sphingosine kinase/sphingosine 1-phosphate (S1P)/S1P receptor axis. Am J Pathol 2012; 181: 85-97.

25. Wang D, Park JS, Chu JS, Krakowski A, Luo K, Chen DJ et al. Proteomic profiling of bone marrow mesenchymal stem cells upon transforming growth factor beta1 stimulation. J Biol Chem 2004; 279: 43725-43734.

26. De Wever O, Mareel M. Role of tissue stroma in cancer cell invasion. J Pathol 2003; 200: 429-447.

27. Hinz B. Formation and function of the myofibroblast during tissue repair. J Invest Dermatol 2007; 127: 526-537.

28. Li H, Fan X, Houghton J. Tumor microenvironment: the role of the tumor stroma in cancer. J Cell Biochem 2007; 101: 805-815.

29. Kono $Y$, Nishiuma T, Nishimura $Y$, Kotani $Y$, Okada T, Nakamura $S$ et al. Sphingosine kinase 1 regulates differentiation of human and mouse lung fibroblasts mediated by TGF-beta1. Am J Respir Cell Mol Biol 2007; 37: 395-404.

30. Gellings Lowe N, Swaney JS, Moreno KM, Sabbadini RA. Sphingosine-1-phosphate and sphingosine kinase are critical for transforming growth factor-beta-stimulated collagen production by cardiac fibroblasts. Cardiovasc Res 2009; 82: 303-312.

31. Cencetti F, Bernacchioni C, Nincheri P, Donati C, Bruni P. Transforming growth factor-beta1 induces transdifferentiation of myoblasts into myofibroblasts via up-regulation of sphingosine kinase-1/S1P3 axis. Mol Biol Cell 2010; 21: 1111-1124.

32. Kinner B, Zaleskas JM, Spector M. Regulation of smooth muscle actin expression and contraction in adult human mesenchymal stem cells. Exp Cell Res 2002; 278: 72-83

33. Jeon ES, Moon HJ, Lee MJ, Song HY, Kim YM, Cho M et al. Cancer-derived lysophosphatidic acid stimulates differentiation of human mesenchymal stem cells to myofibroblast-like cells. Stem Cells 2008; 26: 789-797.

34. Cohen S, Tyrrell DA, Smith AP. Psychological stress and susceptibility to the common cold N Engl J Med 1991; 325: 606-612.

35. Sanchez-Resendis O, Medina AC, Serafin N, Prado-Alcala RA, Roozendaal B, Quirarte GL. Glucocorticoid-cholinergic interactions in the dorsal striatum in memory consolidation of inhibitory avoidance training. Front Behav Neurosci 2012; 6: 33.

36. Chiba S, Numakawa T, Ninomiya M, Richards MC, Wakabayashi C, Kunugi $H$ Chronic restraint stress causes anxiety- and depression-like behaviors, downregulates glucocorticoid receptor expression, and attenuates glutamate release induced by brain-derived neurotrophic factor in the prefrontal cortex. Prog Neuropsychopharmacol Biol Psychiatry 2012; 39: 112-119. 
37. Feldman BJ. Glucocorticoids influence on mesenchymal stem cells and implications for metabolic disease. Pediatr Res 2009; 65: 249-251.

38. Wynn TA. Common and unique mechanisms regulate fibrosis in various fibroproliferative diseases. J Clin Invest 2007; 117: 524-529.

39. Schnabl B, Scholten D, Brenner DA. What is the potential role of antifibrotic agents for the treatment of liver disease? Nat Clin Pract Gastroenterol Hepatol 2008; 5: 496-497.

40. Tomasek JJ, Gabbiani G, Hinz B, Chaponnier C, Brown RA. Myofibroblasts and mechanoregulation of connective tissue remodelling. Nat Rev Mol Cell Biol 2002; 3: 349-363.

41. Guyot C, Lepreux S, Combe C, Doudnikoff E, Bioulac-Sage P, Balabaud C et al. Hepatic fibrosis and cirrhosis: the (myo)fibroblastic cell subpopulations involved. Int J Biochem Cell Biol 2006; 38: 135-151.

42. Gressner AM, Weiskirchen R. Modern pathogenetic concepts of liver fibrosis suggest stellate cells and TGF-beta as major players and therapeutic targets. J Cell Mol Med 2006; 10: 76-99.

43. Ramadori G, Saile B. Portal tract fibrogenesis in the liver. Lab Invest 2004; 84: 153-159.

44. Lieber CS. Alcoholic liver disease: new insights in pathogenesis lead to new treatments. J Hepatol 2000; 32(1 Suppl): 113-128.

45. Iredale JP. Models of liver fibrosis: exploring the dynamic nature of inflammation and repair in a solid organ. J Clin Invest 2007; 117: 539-548.

46. Kisseleva T, Brenner DA. Anti-fibrogenic strategies and the regression of fibrosis. Best Pract Res Clin Gastroenterol 2011; 25: 305-317.

47. Iwaisako K, Brenner DA, Kisseleva T. What's new in liver fibrosis? The origin of myofibroblasts in liver fibrosis. J Gastroenterol Hepatol 2012; 27(Suppl 2): 65-68.

48. Bucala R, Spiegel LA, Chesney J, Hogan M, Cerami A. Circulating fibrocytes define a new leukocyte subpopulation that mediates tissue repair. Mol Med 1994; 1: 71-81.

49. Fausther M, Lavoie EG, Dranoff JA. Contribution of myofibroblasts of different origins to liver fibrosis. Curr Pathobiol Rep 2013; 1: 225-230.

50. Hinz B, Phan SH, Thannickal VJ, Prunotto $M$, Desmouliere A, Varga $J$ et al. Recent developments in myofibroblast biology: paradigms for connective tissue remodeling. Am J Pathol 2012; 180: 1340-1355.
51. Hardy R, Cooper MS. Glucocorticoid-induced osteoporosis-a disorder of mesenchymal stromal cells? Front Endocrinol (Lausanne) 2011; 2: 24.

52. Zhu H, Guo ZK, Jiang XX, Li H, Wang XY, Yao HY et al. A protocol for isolation and culture of mesenchymal stem cells from mouse compact bone. Nat Protoc 2010; 5: 550-560.

53. Hou J, Han ZP, Jing YY, Yang X, Zhang SS, Sun K et al. Autophagy prevents irradiation injury and maintains stemness through decreasing ROS generation in mesenchymal stem cells. Cell Death Dis 2013; 4: e844.

54. Sheridan JF, Feng NG, Bonneau RH, Allen CM, Huneycutt BS, Glaser R. Restraint stress differentially affects anti-viral cellular and humoral immune responses in mice. J Neuroimmunol 1991; 31: 245-255.

55. Lamkin DM, Sloan EK, Patel AJ, Chiang BS, Pimentel MA, Ma JC et al. Chronic stress enhances progression of acute lymphoblastic leukemia via beta-adrenergic signaling. Brain Behav Immun 2012; 26: 635-641.

56. Anglen CS, Truckenmiller ME, Schell TD, Bonneau RH. The dual role of CD8 + $T$ lymphocytes in the development of stress-induced herpes simplex encephalitis J Neuroimmunol 2003; 140: 13-27.

(c) (i) (2)(2) Cell Death and Disease is an open-access journal published by Nature Publishing Group. This work is licensed under a Creative Commons Attribution-NonCommercialShareAlike 3.0 Unported License. The images or other third party material in this article are included in the article's Creative Commons license, unless indicated otherwise in the credit line; if the material is not included under the Creative Commons license, users will need to obtain permission from the license holder to reproduce the material. To view a copy of this license, visit http://creativecommons.org/licenses/ by-nc-sa/3.0/

Supplementary Information accompanies this paper on Cell Death and Disease website (http://www.nature.com/cddis) 